\title{
IN

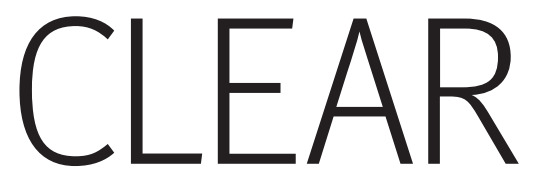 \\ SIGHT
}

\section{Despite measures to improve transparency of research funding, Marisa de Andrade finds there is still scope for confusion}

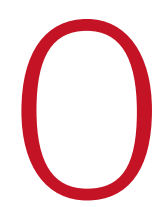
$\mathrm{n}$ a Friday night in March 2009, I joined more than 30 doctors gathered at the Gleneagles Hotel for predinner drinks. We were attending a weekend meeting at the invitation of the University of Dundee's Hypertension Research Centre and Medicines Monitoring Unit after "positive feedback" from a similar gathering in January. Both meetings aimed to provide practices with sufficient information about the Standard Care versus Celecoxib Outcome Trial (SCOT) to enable general practitioners to decide whether to participate.

The invitation did not mention Pfizer, although the drug company was providing $£ 26 \mathrm{~m}$ (€30m; $\$ 43 \mathrm{~m})$ for the study. ${ }^{1}$ Instead, the trial was described as "an academic, investigatorinitiated study, requested by the European Medicines Agency (EMEA) and sponsored by the University of Dundee." The university is working in partnership with the universities of Glasgow, Edinburgh, and Aberdeen and collaborating with researchers from Nottingham University and the University of Southern Denmark. ${ }^{23}$

The SCOT application form submitted to the NHS research ethics committee indicates that Pfizer is the sole funder of the study.

The trial is designed to compare the cardiovascular safety of the cyclo-oxygenase-2 (COX-2) inhibitor celecoxib with that of other non-steroidal anti-inflammatory drugs (NSAID) in men and women who are over 60 , who already take a non-selective NSAID regularly, and who do not have established cardiovascular or peripheral vascular disease or severe heart failure. ${ }^{3}$

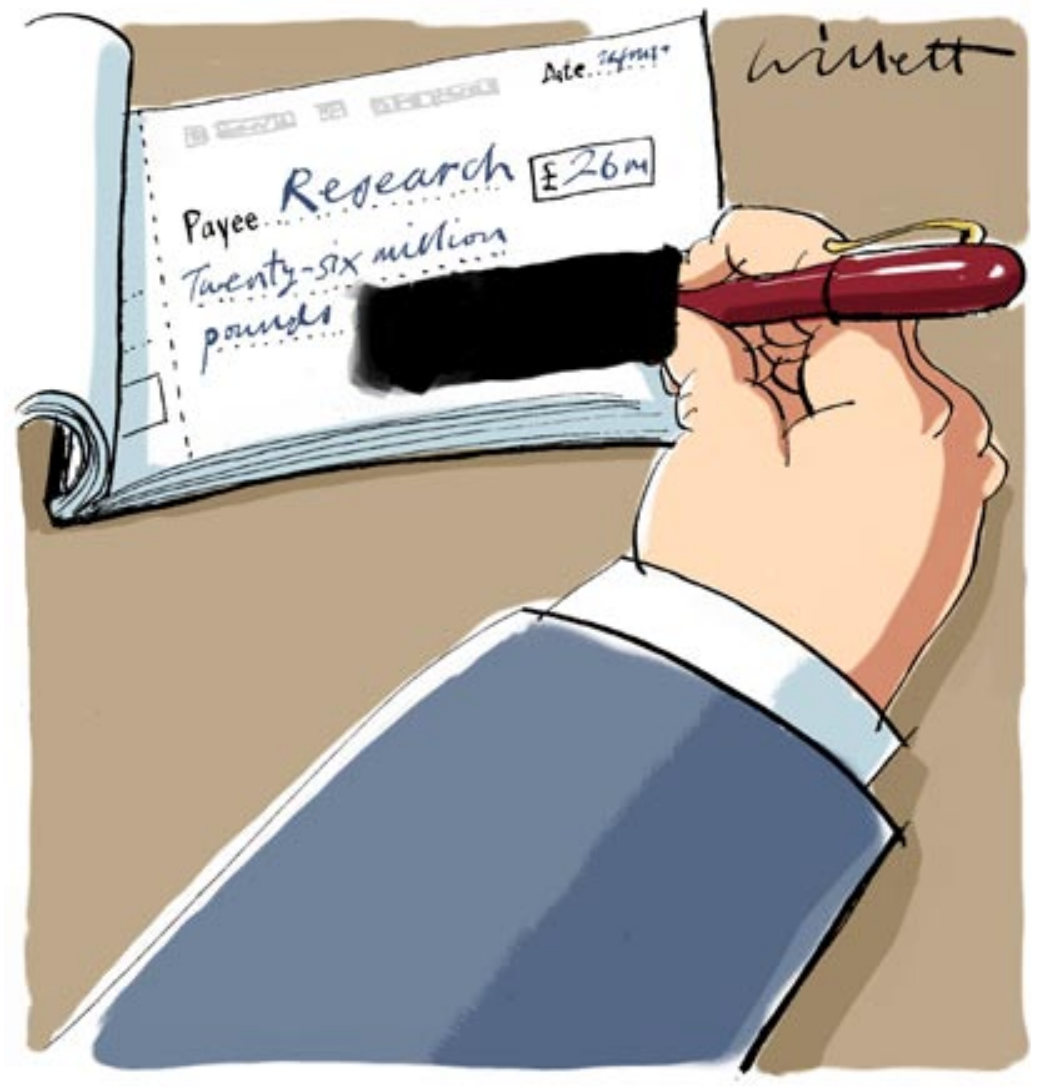

only "standard set menus and no excessive hospitality was given." He also stressed "GPs had given up their Saturday without pay to be trained in trial methodology."

The educational meeting at Gleneagles was a cost cutting measure, according to Professor MacDonald: "We found that if we rented out a room somewhere during the week, doctors weren't coming. But they are coming if we set up meetings at the weekend at Gleneagles. This still works out better for us. The whole deal we get from the hotel is a lot less than $£ 300$. You could say the recession's helped us do the study."

His argument is that doctors have to be paid a locum fee of $£ 350$ a day if these meetings are held during the week and one partner would have to leave the surgery.

Thirty five doctors

Recruitment started in February 2008 and is expected to last three years with a total of 16000 patients. ${ }^{1}$ Dundee University plans to increase the number of participants by running the trial in England and another European country, possibly the Netherlands.

After hearing about the meeting and approaching the university for comment, I received a call from Beattie Communications, the public relations firm that is managing the trial and which lists Pfizer as one of its clients, ${ }^{4}$ and was invited to attend by the chief investigator.

Doctors attending the information meeting, which started at 9 am on Saturday and ended with a three course lunch, had complimentary drinks and dinner the night before, accommodation at the five star luxury hotel on the Friday night, and their travel reimbursed.

The principal investigator, Tom MacDonald, said that attendees received attended the meeting from 25 practices. Up to four GPs were in attendance from a single practice. Some doctors said their practice had already signed up to the trial. One of them admitted coming along just for the hospitality. Another joked, "If we don't sign up now, does that mean we get to come to Gleneagles again and again until we make our minds up?"

Practices that sign up get $£ 1000$ and a further $£ 5$ every two months for each patient reporting progress on a web portal. Professor MacDonald said: "Some practices have more than 50 patients. That's quite a lot of money, but it goes to the practice. Dundee University does not sign any cheques for doctors."

The chief investigator also defends the study's independence: "The SCOT trial is creating vital research capacity. It is entirely run by the University of Dundee, with no pharmaceutical company involvement in 
any of our meetings. As such, mention of the Association of the British Pharmaceutical (ABPI) Code of Practice, which governs how drugs are commercially promoted to doctors by industry, is entirely inappropriate."

Clause 19.1 of the code indicates that meetings with clear educational content should be held at appropriate venues, conducive to the main purpose of the meeting. Lavish, extravagant, or deluxe venues must not be used and companies should avoid using venues that are renowned for their entertainment facilities. As a guide, the ABPI asks the industry to apply the question, "would you and your company be willing to have these arrangements generally known?" when determining whether the arrangements for any meeting are acceptable.

A spokesperson for Pfizer supported Professor MacDonald in his position that the ABPI code did not apply to the Gleneagles meeting: "All meetings organised by Pfizer or on our behalf are conducted to the highest standards of compliance with the ABPI code of practice. The SCOT study is an investigator driven research project led by Professor Tom MacDonald of the University of Dundee, which is the sponsor of the clinical trial. Pfizer has provided financial support to the study, but it is managed and operated independently of Pfizer. This meeting was not organised by Pfizer or on Pfizer's behalf and it was solely the initiative and responsibility of Professor MacDonald and the University of Dundee."

Des Spence is from No Free Lunch UK, an organisation campaigning for a public register of all hospitality and payments received by health professionals. He said: "This is

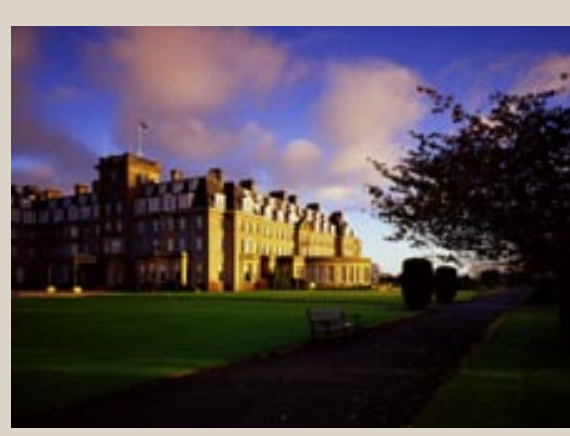

"We found if we rented out a room somewhere during the week, doctors weren't coming. But they are coming if we set up meetings at the weekend at Gleneagles" Tom MacDonald obviously not how patients for trials should be recruited. Doctors should be encouraged to recruit in a trial because they think it's a good thing and will be beneficial for the patient. There are loads of ways they could go about recruiting for trials-they could go to health centres, have lunch meetings, for example-Gleneagles would seem inappropriate to most people. I would also question whether the overnight stay is necessary. Most doctors live in the central belt of Scotland and could drive through for the meeting in the morning."

Yoon Loke, a co-convenor of the Cochrane Adverse Effects Methods Group and senior lecturer in clinical pharmacology at the University of East Anglia, also has reservations: "Like all academic research projects with external funding, Pfizer has agreed to provide a certain sum of money to pay for the trial, and this will include costs of recruitment and investigators' meetings-in Gleneagles for this particular study. The money has been given to Dundee, but the source is still commercial."

He thinks there are cheaper ways to conduct research in the public interest: "My colleagues and I currently have UK Medical Research Council (MRC) funding for a project. When we applied for the money, we put down a sum of $£ 1000$ for meetings, and the MRC agreed to it. The last meeting for 12 researchers cost us roughly $£ 300$-we had sandwiches in a small hotel right next to Peterborough railway station.”

There are lavish meetings and frugalusually tax payer funded-ones, he says. "Tom MacDonald was only able to hold it at Gleneagles because money was coming

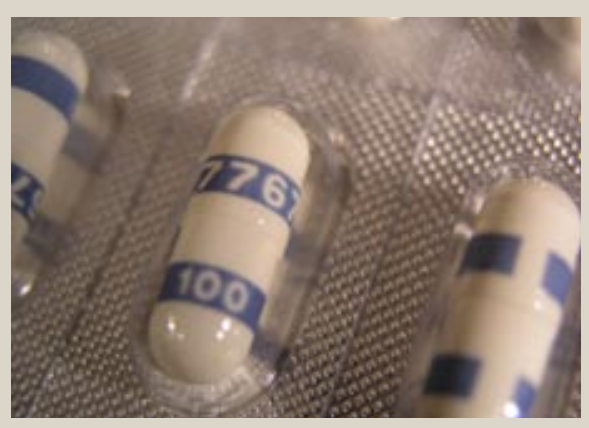

The trial is designed to compare the cardiovascular safety of the cyclo-oxygenase-2 (COX-2) inhibitor celecoxib with that of other non-steroidal anti-inflammatory drugs (NSAIDs) from Pfizer. There would be no chance of Dundee University agreeing to pay for such a meeting from university funds."

\section{Appropriate design?}

Dr Yoke, who specialises in developing methods for evaluating data on adverse effects, is also concerned about the trial's design: "The patients all undergo a run-in phase before randomisation where they take celecoxib for two weeks prior to being allowed to take full part in the trial. ${ }^{3}$ What is the effect of this run-in phase? In my opinion, all the people who suffer side effects from celecoxib will drop out in the first two weeks, thus ensuring that the only people who continue in the trial will be those who do well with celecoxib. I have strong concerns about this type of study design as I don't believe that the safety data would be as valid as with other ones."

Robert M Elashoff, professor of biostatistics and biomathematics at the University of California, who specialises in clinical trials design and analysis, agrees: "I think the effect of the run-in is to remove patients with unfavorable cardiovascular or gastrointestinal response. Those with side effects to celecoxib will be out of the study.

"Using a run-in with so many studies with celecoxib completed is silly. SCOT should be revised and the run-in deleted."

A spokesperson for Pfizer said: "The SCOT study is an investigator driven research project ... While Pfizer has provided financial support to the study, it is managed and operated independently of Pfizer. Therefore, the study sponsors should be contacted for a response to questions relating to the conduct of this study."

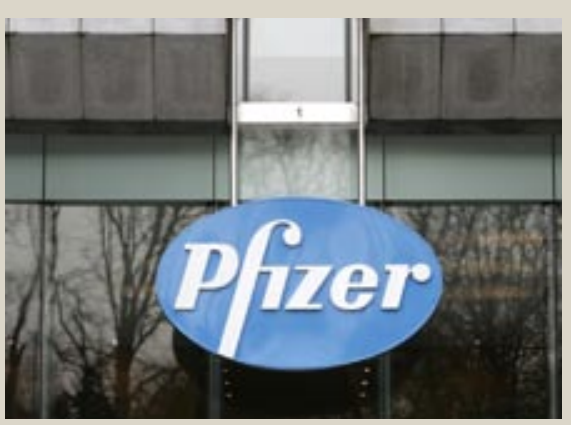

Pfizer has provided financial support to the study, but it is managed and operated independently of Pfizer 
The University of Dundee has declined to comment on these specific criticisms of the trial design but has released the full protocol after a request under the Freedom of Information Act. The document provides a rationale behind choices of study design: "The trial identifies chronic NSAID users in the population who were not taking 'coxib' [COX-2] drugs. These subjects have demonstrated tolerance to NSAIDs. Switching of drug therapy to celecoxib as would happen to $50 \%$ of subjects if randomization occurred without an open label phase was thought to introduce a bias in that subjects would be more likely to tolerate their previous drug than the new one. For this reason the open label phase allows those who have relatively similar tolerability and efficacy to both therapies prior to randomization."

The document explains that at the end of the run-in period, "Subjects who have taken at least one dose of celecoxib and who do not express a strong preference for either their previous treatment or celecoxib will be eligible for randomisation. Preference will be determined by the patient response to a questionnaire."

\section{Transparency}

The trial has been registered on ClinicalTrials. gov so that the data can be used in the US. ${ }^{3}$ The register encourages transparency in clinical research by providing free access to information about a trial's funding, sponsorship, methodology, intervention, and research question. ${ }^{5}$ Its policy is consistent with US law and does not require the listing of collaborators or funders if they are not considered the sponsor. $^{6}$

There is no mention of Pfizer in the SCOT trial registration form. ${ }^{3}$ This isn't a legal requirement, but Deborah Zarin, director of ClinicalTrials.gov, said that it may be a problem for other agencies: "It is up to the registrant and whomever enforces the other policies to ensure compliance. We don't enforce their policies, but they have chosen to use our site as the mechanism for complying with their laws."

A spokesperson for the International Committee of Medical Journal Editors (ICMJE) said: "As stated in the ICMJE policy, ${ }^{7}$ funding source or sponsor is a required field for registration. Without this information, the ICMJE would consider registration insufficient."
A spokesperson for Pfizer said: "Pfizer considers investigator driven research to be important in advancing disease treatments and consequently improving the lives of patients. Pfizer encourages all investigators to disclose information on research they are conducting; however, there is no formal requirement for them to do so."

The University of Dundee has not commented on why it chose to leave out the funding source from the clinical register.

David Miller, professor of sociology at Strathclyde University, has also raised concerns that the SCOT website (www. scottrial.co.uk) doesn't mention funding from Pfizer-a fact also missing from some news pieces announcing the study. ${ }^{8-10}$ "Neglecting to mention the financial sponsor of the research is deceptive," he says. "On the other hand the recruitment of doctors via entertainment in five star luxury also appears to be ethically questionable."

Howard Brody, director of the Institute for the Medical Humanities at the University of Texas Medical Branch, specialises in ethical issues in primary care and professional integrity in clinical research. He added: "The 'purpose' of the study in the trial register reads more like a press release promoting celecoxib than a statement of today's science. The notion that other NSAIDs pose a significant cardiovascular risk, comparable to that of COX-2 drugs, is a very dubious claim. This certainly makes me worried that the information to be presented to research subjects will sound more like a marketing ploy and less like an assessment of the science."

The participant information sheet presented to potential research subjects states that "one NSAID which appears to be at least as safe as most NSAIDs and may be safer than some is celecoxib." The document highlights that "there have also been a number of recent studies of this group of drugs [COX-2s] some of which have suggested there may be a link between these newer drugs and increased heart disease and strokes. For Celebrex [celecoxib], this evidence is not conclusive and there have been many studies that have shown no increased risk of heart disease and strokes." It points to a recent meta-analysis suggesting that cardiovascular effects for celecoxib are similar to those of other NSAIDs and states "there is also evidence that older NSAIDs have cardiovascular effects."
Professor MacDonald insists: "This isn't a commercially viable trial for Pfizer. It's not going to help their business model. They're doing this because they have to fill a regulatory EMEA commitment."

The health regulator has been monitoring COX-2 inhibitors since 2004, when refecoxib was withdrawn because of a risk of thrombotic cardiovascular events and questions were raised regarding the cardiovascular safety of other COX-2 inhibitors. ${ }^{11}$

As part of the EMEA's December 2005 decision to keep celecoxib on the market, it recommended a long term study to investigate its safety relative to non-selective NSAIDs.

An EMEA spokesperson said: "You cannot force anyone to conduct clinical trials, but if a company wants its product to stay on the market then we need to be convinced that it should be there. It is in Pfizer's commercial interest to do it."

Professor MacDonald insists it is "Scottish Government policy to promote research in primary care," and the trial is "fully endorsed by the chief medical officer, the chief pharmaceutical officer and the chief scientist for Scotland [and] uses methodology which hopefully will allow similar types of medicines research to be done more easily in the future."

Marisa de Andrade freelance journalist

marisa.de-andrade@strath.ac.uk

Competing interests: MdA is the editor of the Pharma portal on SpinProfiles - an online database of people, issues and groups shaping the public agenda.

1 Edinburgh Clinical Trials Unit. SCOT (Standard Care versus Celecoxib Outcome Trial). July 2009. www.clinicaltrials. ed.ac.uk/trials/scot/default.asp.

2 Pharma Marketletter. SCOT trial to compare Celebrex' safety with that of older NSAIDs. 19 Dec, 2007. http:// www.scottrial.co.uk/p191207.htm.

3 Clinical Trials.gov. The Standard Care versus Celecoxib Outcome Trial (SCOTLSSS). 2007. http://clinicaltrials gov/ct2/show/NCT00447759.

4 Beattie Communications. A full service healthcare PR offering. July 2009. www. beattiegroup.com/ healthcarepr/healthcare-pr-services.aspx.

5 Smyth RL. Making information about clinical trials publicly available. BMJ 2009;338:b2473.

6 ClinicalTrials.gov. Protocol data element definitions. 2008. http://prsinfo.clinicaltrials.gov/definitions.html.

7 International Committee of Medical Journal Authors. Uniform requirements for manuscripts submitted to biomedical journals: writing and editing for biomedical publication. 2008. www.icmje.org/

8 Scotland is set to lead the way in a $£ 26$ million drug trial. Daily Mail 2007Dec 17. www.scottrial.co.uk/ p171207b.htm.

9 Arthritis sufferers benefit from $£ 26 \mathrm{~m}$ trial in Scotland. Herald 2007Dec 17. www.scottrial.co.uk/p171207d.htm

10 New study to test arthritis drugs. BBC News 2007Dec 17. http://news.bbc.co.uk/2/hi/uk_news/scotland/ tayside_and_central/7148100.stm.

11 European Medicines Evaluation Agency. Human medicines-referrals. 2005. www.emea.europa.eu/ htms/human/referral/article31/celecoxib_05.htm.

Cite this as: BMJ 2009;339:b3443 